Article available at nttp://Www.parasite-journal.org or nttp://dx.dol.org/10.1051/parasite/1998053285

\title{
L'ÉCHINOCOCCOSE ALVÉOLAIRE DANS LE DÉPARTEMENT FRANÇAIS DES ARDENNES : CAS ISOLÉS OU NOUVEAU FOYER?
}

\author{
DEPAQUIT J.*, GALLEGO A.**, USSEGLIO F.***, LIANCE M.**** \& FAVRIEL J.M.**
}

Summary : ALVEOLAR ECHINOCOCCOSIS IN THE ARDENNES AREA (FRANCE) : ISOLATE CASES OR NEW FOCUS?

The first three autochtonous cases of alveolar echinococcosis were diagnosed in the Ardennes area (France). This is the most occidental localization of this disease in Northern Europe. The authors discuss these cases with an epidemiological regard. They are looking for relationships with natural parasitic cycle in the neighbouring country Belgium and their consequences on local public health in the future.

KEY WORDS : Alveolar echinococcosis, epidemiology, human cases, France, Ardennes.

\section{Résumé :}

Les trois premiers cas humains autochtones d'échinococcose alvéolaire ont été diagnostiqués dans le département des Ardennes (France). Ils constituent les mentions les plus occidentales de la maladie en Europe septentrionale. Les auteurs discutent ces cas dans un contexte épidémiologique et évoquent un lien probable avec les cycles connus régionalement en milieu naturel et ses conséquences sur la santé publique locale dans les années à venir

MOTS CLÉS : Alveolar echinococcosis, epidemiology, human cases, France, Ardennes.

hépatique. L'hyperéosinophilie (2 464 éléments $/ \mathrm{mm}^{3}$ ) oriente initialement le diagnostic vers une fasciolose mais le bilan parasitaire s'avère négatif. A l'occasion d'une récidive de l'ictère (juin 1997), un cathétérisme rétrograde évoque un cancer du hile. L'amaigrissement est cette fois, de $25 \mathrm{~kg}$ en 7 mois. L'hyperéosinophilie persiste toujours $\left(500\right.$ éléments $/ \mathrm{mm}^{3}$ ). Le diagnostic macroscopique d'échinococcose alvéolaire est posé en per-opératoire. Le chirurgien pratique une exérèse aussi complète que possible. L'examen anatomopathologique confirme l'étiologie ainsi que la sérologie effectuée par ELISA, plus fortement positive vis-à-vis d'un extrait antigénique total d'Echinococcus multilocularis que vis-à-vis des antigènes de liquide hydatique. Le patient décède un mois plus tard de complications infectieuses.

Ce cas est en fait le troisième diagnostiqué au Centre Hospitalier de Charleville-Mézières où une enquête rétrospective menée sur 15 ans nous a permis de retrouver 2 autres cas.

Le premier est celui d'un patient opéré d'urgence (juillet 1984) pour rupture d'un kyste hydatique intrapéritonéal. Une sérologie positive en hémagglutination et en immunoéléctrophorèse vis à vis d'un antigène d'E. granulosus impute initialement la pathologie à cette espèce. Cependant, de nombreuses récidives orientent le diagnostic vers une échinococcose alvéolaire qui sera confirmé par l'examen anatomopathologique des prélèvements hépatiques. Le patient décède

en 1990.

\footnotetext{
* Laboratoire de Parasitologie, Faculté de Pharmacie, 51, rue Cognacq-Jay, F-51096 Reims cedex.

** Service de Gastro-entérologie, Hôpital Manchester, F-08000 Charleville-Mézières.

*** Service de Médecine, Centre Hospitalier, F-08200 Sedan.

**** Laboratoire de Parasitologie, Faculté de Médecine, F-94010 Créteil.

Correspondance : Jérôme Depaquit : Tél. : 0326053723 - Fax

0326053597.
} 
Le second cas est celui d'un patient opéré en 1985 pour exérèse d'une tumeur kystique hépatique. L'examen anatomopathologique révèle le diagnostic d'échinococcose alvéolaire. Suite à de nombreuses récidives, le patient subit une transplantation hépatique orthotopique en 1992. Il décédera en 1995.

\section{COMMENTAIRES ÉPIDÉMIOLOGIQUES}

L es trois patients étaient des ruraux sédentaires, ce qui suggère une transmission locale de la maladie. Ils habitaient trois villages distants de quelques dizaines de kilomètres : Etalle, Fagnon et Messincourt (fig. 1). Ces localités sont situées dans des zones vallonnées mixtes (bois et champs) typiques des contreforts du massif Ardennais.

L'existence de ces trois cas cliniques vient à l'appui de l'hypothèse de l'extension de l'aire de répartition de la maladie en Europe. Dans la Belgique voisine (province de Luxembourg), Brochier et al. (1992) notent pour la première fois l'existence du parasite à l'occasion d'une prospection réalisée chez des renards. La prévalence observée est alors de 17,5\%. Ces résultats sont confirmés par Losson et al. (1997) qui trouvent $51 \%$ des renards porteurs d'Echinococcus multilocularis. Cette prévalence très élevée égale ou dépasse celles rencontrées dans les foyers endémiques français (Pétavy et al., 1990) ou étrangers (Giraudoux, 1991). Aux Pays-Bas, Van der Giessen et al. (1997) observent également un portage du parasite par le renard. Ces résultats sont toutefois à analyser avec prudence dans la mesure où la prévalence du portage vulpin du parasite varie beaucoup d'une année à une autre.

En France, l'utilisation de la vaccination par voie orale des renards prévaut depuis quelques années sur leur abattage dans le cadre de la lutte contre la rage. D'autre part, l'adoucissement des conditions météorologiques hivernales observé depuis quelques années a entraîné une pullulation des rongeurs. De ces deux facteurs, résulte un accroissement très significatif des populations vulpines qui est confirmé par une multiplication par 10 des indices kilométriques d'abondance des renards dans le département des Ardennes entre 1986 et 1996 (source : entente interdépartementale de lutte contre la rage). Les renards réoccupent une niche écologique vidée par la lutte intensive menée contre eux durant les années 1980 et permettent ainsi la réalisation locale du cycle sauvage, préalable nécessaire à toute infestation humaine.

\section{CONCLUSION}

i aucun cas humain n'a encore jamais été rapporté en Belgique (Carlier, U.L.B. Bruxelles, communication personnelle), la présence d'un foyer sauvage actif dans ce pays fait planer le risque d'apparition d'autres cas humains sur le massif Ardennais tout entier. En 1977, Rey et al. ont révélé l'exis-

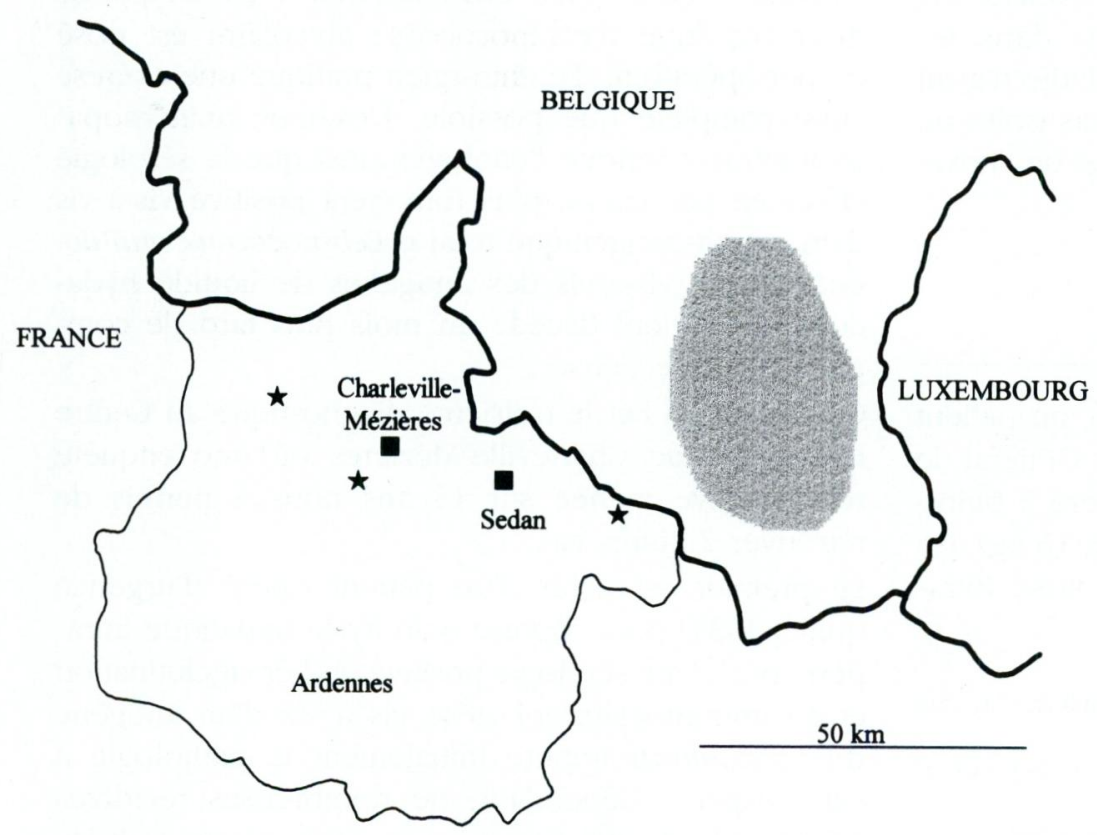

Fig. 1. - Localisation des cas humains d'échinococcose alvéolaire dans le département des Ardennes. 
tence du foyer auvergnat d'échinococcose alvéolaire par l'observation de deux cas cliniques régionaux. Dans la mesure où le délai entre l'infestation par Echinococcus multilocularis et les symptômes cliniques de la maladie varie en moyenne entre cinq et dix ans, chaque cas humain reflète une situation épidémiologique antérieure de quelques années et l'inquiétude de voir flamber un foyer d'échinococcose alvéolaire dans cette région est légitime si l'on considère comme probable la corrélation entre le développement du cycle parasitaire et l'accroissement très significatif des populations vulpines dans les Ardennes.

\section{BIBLIOGRAPHIE}

Bresson-Hadni S., Laplante J.J., Lenys D., Rohmer P., GottStein B., Jacquier P., Mercet P., Meyer J.P., Miguet J.P. \& VuitTon D.A. Seroepidemiologic screening of Echinococcus multilocularis infection in a european area endemic for alveolar echinococcosis. American Journal of Tropical Medicine and Hygiene, 1994, 51, 837-846.

Brochier B., Coppens P., Losson B., Aubert M.F.A., Bauduin B., Barrrat M.J., Costy F., Perharpre D., Pouplard L. \& PASTORET P.P. Enquête sur l'infestation du renard roux (Vulpes vulpes), par Echinococcus multilocularis en province de Luxembourg (Belgique). Annales de Médecine Vétérinaire, 1992, 136, 497-501.

Giraudoux P. Utilisation de l'espace par les hôtes du ténia multiloculaire (Echinococcus multilocularis) : conséquences épidémiologiques. Thèse de Sciences Biologiques, Dijon, 1991.

Houin R. L'échinococcose alvéolaire. L'eurobiologiste, 1996, 30, 9-14.

Losson B., Mignon B., Brochier B., Bauduin B. \& Pastoret P.P. Infestation du renard roux (Vulpes vulpes) par Echinococcus multilocularis dans la province de Luxembourg (Belgique) : résultats de l'enquête effectuée entre 1993 et 1995. Annales de Médecine Vétérinaire, 1997, 141, 149-153.

Petavy A.F., Deblock S. \& Prost C. Epidémiologie de l'échinococcose alvéolaire en France. I. Helminthes intestinaux du renard commun (Vulpes vulpes) en Haute-Savoie. Annales de Parasitologie Humaine et Comparée, 1990, 65, 22-27.

Rey M., Morin B., Petavy A.F., Cambon M. \& Baril A. Premières observations auvergnates d'échinococcose alvéolaire. La Nouvelle Presse Médicale, 1977, 6, 4070-4071.

VAN der Giessen J., Franchimont H., Rombouts Y., Limper L. \& HOMAN W. Presence of Echinococcus multilocularis in the Netherlands. Archivos Internacionales de la Hidatidosis, vol XXXII, International Association of Hydatidology (Ed), Lisbonne, Portugal, 1997, 242-243.

Reçu le 19 décembre 1997 Accepté le 11 février 1998 